\title{
A Multiplicity Theorem for Representations of Inhomogeneous Compact Groups
}

\author{
H. D. Doebner and J. TolaR ${ }^{\star}$ \\ International Centre for Theoretical Physics, Trieste, Italy, \\ and the University of Marburg, Fed. Rep. Germany
}

Received August 14, 1970

\begin{abstract}
The problem of finiteness of multiplicities of irreducible unitary representations of a compact subgroup is considered for decompositions of irreducible unitary representations of locally compact groups. A simple solution is found for inhomogeneous compact groups and for a physically interesting class of groups with a non-abelian radical.
\end{abstract}

\section{Introduction}

We want to discuss the following problem: let $U(G)$ be any irreducible unitary representation of a Lie group $G$ with $K$ being a maximal compact subgroup of $G$. Decompose $U(G)$ with respect to $K$, i.e., $U(G) \downarrow K$, and ask: for which groups $G$ are the multiplicities of all irreducible unitary representations $U^{[\alpha]}(K)$ in the decomposition of $U(G)$ finite for all $\alpha$, where $\alpha$ labels the irreducible unitary representations of $K$. Non-compact Lie groups $G$ possessing this property are sometimes called "groups which admit a large compact subgroup" (Ref. [1], p. 641).

The decomposition $U(G) \downarrow K$ is needed for physical applications of dynamical groups, which are in general non-compact embeddings $G$ of a compact semi-simple symmetry group $K^{\prime}$ possessing an irreducible unitary representation $U(G)$ such that $U(G) \downarrow K^{\prime}=U_{\text {red }}\left(K^{\prime}\right)$, where $U_{\text {red }}\left(K^{\prime}\right)$ is a given reducible unitary representation of $K^{\prime}$. The simplest embeddings of $K^{\prime}$ are those in which $K^{\prime}$ is isomorphic to the maximal compact subgroup $K$ of $G$.

The simply connected embeddings $G$ can be classified using the LevyMalcev decomposition $G=N \otimes S$, where $N$ and $S$ are simply connected Lie groups, the Levy factor $S$ being semi-simple and the radical $N$ solvable. Because $K^{\prime}$ is semi-simple and compact, it has to be embedded in $S$, $K^{\prime} \subset S$, and we shall distinguish the following cases $\left(T_{n}\right.$ is an $n$-dimensional abelian group):

$\star$ On leave of absence from Faculty of Nuclear Science, Czech Technical University, Prague, Czechoslovakia. 
a) $G=S$,

b) $G=T_{n} \otimes_{\sigma} S$,

c) $G=K \otimes_{\sigma^{\prime}} S$,

where $\sigma$ and $\sigma^{\prime}$ are homomorphisms of $S$ into the automorphism group of $T_{n}$ and $N$ respectively. For non simply connected groups there are more possibilities, which can be classified easily for the connected ones.

We present a new solution of the multiplicity problem for connected Lie groups $G$ with a semi-direct decomposition of type b) and we derive results for special examples of $\mathrm{c}$ ). The usual mathematical technique to do this is to use a group algebra of well behaved functions over $G$. We refer for $G=S$ to papers of Harish-Chandra where he announces [2] and later proves [3] that all connected semi-simple Lie groups with a faithful finite-dimensional representation have large compact subgroups, and to Godement's paper [4] in which this property is proved for $G=T_{n} \otimes_{\sigma} K$. They also derive upper bounds for the multiplicities (see also Ref. [10]).

So a result for the cases a) and b) is already known. However, because the proofs for both a) and b) are rather complicated, it is worthwhile to look for a more direct technique which can also be applied to c). It is the aim of this paper to describe such a method which is based on a lemma by Gel'fand, Graev and Pyatetskii-Shapiro. They analyse the operators

$$
U_{\varphi}=\int_{G} \varphi(g) U(g) d \mu_{G}(g)
$$

for a given continuous unitary representation $U(G)$ of a locally compact group $G$ in a Hilbert space $\mathscr{H} ; d \mu_{G}(g)$ is a right-invariant Haar measure on $G$. Denote by $L(G)$ and $L^{1}(G)$ the spaces spanned by continuous complex functions on $G$ with compact support, and by absolutely integrable complex functions on $G$, respectively. Then $U_{\varphi}$ has the following properties:

Lemma 1. (Ref. [5], Chap. I, Sec. 2.3).

If the operator $U_{\varphi}$ is completely continuous in $\mathscr{H}$ for every $\varphi \in L(G)$, then $\mathscr{H}$ splits into a direct sum of a countable number of G-invariant subspaces $\mathscr{H}^{[\alpha]}$ such that $\mathscr{H}^{[\alpha]}$ carries $m(\alpha)$-times the irreducible unitary representation $U^{[\alpha]}(G)$ with $m(\alpha)$ finite $(m(\alpha) \text { is the multiplicity })^{1}$.

Lemma 2. (Ref. [2]; p. 515 of Ref. [4]; Sec. 8 of Ref. [10]). Let $U(G)$ be an irreducible unitary representation of a locally compact group $G$ in $\mathscr{H}$ and $K$ be a closed compact subgroup of $G$; assume that $\mathscr{H}$ splits into a direct sum of a countable number of $K$-invariant subspaces $\mathscr{H}^{[\alpha]}$ each carrying $m(\alpha)<\infty$ copies of an irreducible unitary representation of $K$; then $U_{\varphi}$ is completely continuous in $\mathscr{H}$ for every $\varphi \in L(G)^{1}$.

1 The assumption of unimodularity of $G$ used only for technical reasons in the cited papers has been dropped. 


\section{Multiplicity Theorem for Inhomogeneous Compact Groups}

1. We start with a

Theorem. Let $G=T \otimes_{\sigma} K$ be a connected semidirect product of a compact Lie group $K$ and an abelian group ${ }^{2} T$. Then $U(G)$ decomposes into a direct sum of irreducible unitary representations $U^{[\alpha]}(K)$ labelled by $\alpha$,

$$
U(G) \downarrow K=\bigoplus_{\alpha} m(\alpha) U^{[\alpha]}(K),
$$

where all the multiplicities $m(\alpha)$ are finite.

2. For a proof we need a representation theory for $G$, which is well known $[1,6]$. Since the semidirect product $G$ is regular and unimodular, any $U(G)$ can be constructed as induced representation ${ }^{3} U^{L}(G)$ in a Hilbert space $\mathscr{H}^{L}$ of vector-valued functions $f$ over $G$ via

and

$$
f(h g)=L(h) f(g) \quad(h \in H, g \in G)
$$

$$
\left[U^{L}(g) f\right]\left(g_{1}\right)=f\left(g_{1} g\right) \quad\left(g, g_{1} \in G\right) .
$$

Here $L(H)$ is an irreducible unitary representation of $H=T \otimes K_{0}, K_{0}$ is a stationary subgroup of some $K$-orbit in the character space $\hat{T}$ of $T$. The elements $h \in H$ can be written as $h=t k_{0}, t \in T, k_{0} \in K_{0} ; L(H)$ has the form

$$
L(h)=\chi(t) D\left(k_{0}\right)
$$

with $D\left(K_{0}\right)$ being a finite-dimensional irreducible unitary representation of $K_{0}$ and $\chi \in \hat{T}$. The norm in $\mathscr{H}^{L}$ is

$$
\|f\|^{2}=(f, f)=\int_{X}\langle f, f\rangle_{L}(x) d \mu_{X}(x) ;
$$

$\langle f, f\rangle_{L}$ is the scalar product in the representation space of $L(H) ; d \mu_{X}(x)$ is the right-invariant measure on the homogeneous space $X \approx G / H$.

3. Now we are prepared to prove the theorem. Restrict the representation $U^{L}$ to the maximal compact subgroup $K$ of $G$, i.e., $U^{L} \downarrow K \equiv U(K)$ and apply Lemma 1 to $U(K)$. Because of the isomorphism $X \approx G / H$ $\approx K / K_{0}$, the functions $f \in \mathscr{H}^{L}$ are defined on $X$, or on a Borel set $\Lambda \in K$ intersecting each coset from $K / K_{0}$ just in one point $k_{x} \in \Lambda$ in one-one correspondence with $x \in X$. Hence we obtain a Hilbert space $\mathscr{H}^{L}$ of vector-valued functions over $K$ with

$$
f\left(k_{0} k\right)=D\left(k_{0}\right) f(k) \quad\left(k_{0} \in K_{0}, k \in K\right) .
$$

${ }^{2}$ Locally compact and separable; the index indicating the dimension is dropped.

${ }^{3}$ Representations of $G=T \times K$ are of type I (see Ref. [7] and Ref. [6], pages 52, 57, and 178). 
$U(K)$ is defined via

$$
[U(k) f]\left(k_{1}\right)=f\left(k_{1} k\right) \quad\left(k_{1} \in \Lambda\right) .
$$

Let us calculate now

$$
\begin{aligned}
{\left[U_{\varphi} f\right]\left(k_{1}\right) } & =\int_{K} \varphi(k)[U(k) f]\left(k_{1}\right) d \mu_{K}(k)=\int_{K} \varphi(k) f\left(k_{1} k\right) d \mu_{K}(k) \\
& =\int_{K} \varphi\left(k_{1}^{-1} k^{\prime}\right) f\left(k^{\prime}\right) d \mu_{K}\left(k^{\prime}\right)
\end{aligned}
$$

where $k^{\prime}=k_{1} k$ and the left invariance of $\mu_{K}$ has been used. Any $k^{\prime} \in K$ can be factorized as

and

$$
k^{\prime}=k_{0} k_{2} \quad\left(k_{0} \in K_{0}, k_{2} \in \Lambda\right),
$$

$$
\int_{K} \psi\left(k^{\prime}\right) d \mu_{K}\left(k^{\prime}\right)=\int_{\Lambda} d \mu_{\Lambda}\left(k_{2}\right) \int_{K_{0}} \psi\left(k_{0} k_{2}\right) d \mu_{K_{0}}\left(k_{0}\right)
$$

holds. Then $U_{\varphi}$ can be considered as an integral operator over $\Lambda$,

with kernel

$$
\left[U_{\varphi} f\right]=\int_{A} K\left(k_{1}, k_{2}\right) f\left(k_{2}\right) d \mu_{\Lambda}\left(k_{2}\right),
$$

$$
K\left(k_{1}, k_{2}\right)=\int_{K_{0}} \varphi\left(k_{1}^{-1} k_{0} k_{2}\right) D\left(k_{0}\right) d \mu_{K_{0}}\left(k_{0}\right) .
$$

$K\left(k_{1}, k_{2}\right)$ is continuous in $k_{1}, k_{2}$, because $\varphi$ and $D$ are continuous. Since the integration in (3) goes over a compact domain $\Lambda, U_{\varphi}$ is completely continuous (see Ref. [7], Secs. 54 and 108) for any continuous function $\varphi(k), k \in K$. So Lemma 1 applies and proves the theorem.

\section{Multiplicities for Groups with Abelian Radical}

1. With the result of Sec. II we have solved type b) for $S$ being compact. For noncompact $S$ the following lemma holds:

Lemma 3. Let $G=T \otimes S$ be a connected semidirect product of an abelian group $T$ and a semisimple noncompact group $S$ with maximal compact subgroup $K$. Then the multiplicities of irreducible representations $U(K)$ occuring in the decomposition of irreducible unitary representations of $G$ in respect to $K$ cannot be all finite ${ }^{4}$.

For a proof it is sufficient to show that $U_{\varphi}^{L}$ is not completely continuous on $\mathscr{H}^{L}$ for at least one absolutely integrable function $\varphi$ on $G$, e.g., for

$$
\varphi_{0}(g)= \begin{cases}1 & (g \in K) \\ 0 & (g \notin K, g \in G) .\end{cases}
$$

To do this, we note that a linear operator is completely continuous if and only if any bounded set $\mathscr{S} \subset \mathscr{H}^{L}$ is mapped by it onto a relatively compact

4 We assume faithful representations of $G$. 
(precompact) set (see Ref. [7], Sec. 133). Taking for $\mathscr{S}$ the unit ball

$$
\mathscr{S}=\left\{f \in \mathscr{H}^{L} \mid f\left(h_{1} k\right)=f\left(h_{1}\right),\|f\| \leqq 1\right\},
$$

we have for an induced representation $U^{L}$ of $G$ that

$$
\left[U_{\varphi_{0}}^{L} f\right]\left(h_{1}\right)=\int_{G} \varphi_{0}(g)\left[U^{L}(g) f\right]\left(h_{1}\right) d \mu_{G}(g)=\int_{K} f\left(h_{1} k\right) d \mu_{G}(k)
$$

maps $\mathscr{S}$ on itself. But $\mathscr{S}$, though bounded, is not precompact since there exists a complete orthonormal system $\left\{f_{n}\right\} \subset \mathscr{S}$ defined on the double coset space $K \backslash H / H_{0}$ such that $\left\|f_{n}\right\|=1$ and $\left\|f_{n}-f_{n^{\prime}}\right\|=\sqrt{2}$ for $n \neq n^{\prime}$.

2. A similar argument can be applied if $G=T \otimes K$ and if $U^{L}(G)$ is restricted to a proper closed subgroup $K^{\prime}$ of $K$.

\section{Multiplicities for Groups with a Non-abelian Radical}

1. Our method cannot be extended to simply connected solvable non-abelian $N$ because it requires the knowledge of all irreducible unitary representations of the groups $G=N \otimes K$. Only for certain types of $N$ is a representation theory at hand. So we will discuss this examples. Take for $N$ the central extension of the $n$-dimensional Heisenberg group $G_{E}(n)$, being a connected real Lie group with the Lie algebra generated by $\mathbb{Q}_{i}, \mathbb{P}_{j}, \mathbb{C}$, satisfying the commutation relations of the Heisenberg algebra

$$
\begin{gathered}
{\left[\mathbb{Q}_{i}, \mathbb{Q}_{j}\right]=\left[\mathbb{P}_{i}, \mathbb{P}_{j}\right]=\left[\mathbb{Q}_{i}, \mathbb{C}\right]=\left[\mathbb{P}_{j}, \mathbb{C}\right]=0, \quad\left[\mathbb{Q}_{i}, \mathbb{P}_{j}\right]=\delta_{i j} \mathbb{C},} \\
i, j=1,2, \ldots, n .
\end{gathered}
$$

The automorphism group of $G_{E}(n)$ is known and so the groups which can be coupled semidirectly to $G_{E}(n)$; e.g., $G=G_{E}(n) \otimes S O(n)$ [8] and $G=G_{E}(8) \otimes S U(3)[9]$ are possible couplings.

2. A representation theory of $G$ can be developed along the lines given in Ref. [8], where only the special case $S=S O(n)$ is treated. The group $G$ can be written as a regular semidirect product $(Q$ and $P$ are $n$-dimensional abelian groups with generators $\mathbb{Q}_{i}$ and $\mathbb{P}_{i}$ respectively).

$$
G=G_{1} \otimes G_{2}, \quad G_{1}=C \otimes Q, \quad G_{2}=P \otimes S .
$$

It is sufficient to show that the multiplicities cannot be finite for the subgroup $G^{\prime}=G_{1} \otimes G_{2}^{\prime}, G_{2}^{\prime}=P \otimes K$ where $K$ is a maximal compact subgroup of $S$.

All irreducible unitary representations $U^{L}\left(G^{\prime}\right)$ of $G^{\prime}$ can be induced from irreducible unitary representations of the subgroup $H=G_{1} \otimes K$ :

$$
h=g_{1} k \rightarrow L(h)=\chi_{m, \boldsymbol{q}}\left(g_{1}\right) D(k) \text {. }
$$


Then the induced representations are defined on the space $\mathscr{H}^{L}$ of vectorfunctions $f$ over $G$ with values in $L(H)$. Assume

$$
f(h g)=L(h) f(g)
$$

then the inner product on $L(H)$ is constant on $X \approx G /\left(G_{1} \otimes K\right)$, and $\mathscr{H}^{L}$ is a Hilbert-space with an inner-product defined with the right invariant measure

$$
d \mu_{X}(\boldsymbol{q})=d^{n} \boldsymbol{q} .
$$

and the representation acts as

$$
\left[U^{L}(g) f\right](\hat{g})=f(\hat{g} g) .
$$

3. For a discussion of the multiplicity we are interested in

$$
\left[U_{\varphi}^{L} f\right](\hat{g})=\int_{G} \varphi(g)\left[U^{L}(g) f\right](\hat{g}) d \mu_{G}(g)=\int_{G} \varphi(g) f(\hat{g} g) d \mu_{G}(g) .
$$

Taking as before

and

$$
\varphi_{0}(g)=\left\{\begin{array}{l}
1 \ldots(g \in K) \\
0 \ldots(g \notin K, g \in G)
\end{array}\right.
$$

$$
\mathscr{S}=\left\{f \in \mathscr{H}^{L} \mid f(\hat{g} k)=f(\hat{g}),\|f\| \leqq 1\right\},
$$

i.e., a unit ball in the Hilbert space of functions over $K \backslash G / G_{1} \otimes K$, we find that $U_{\varphi}$ is not completely continuous on $\mathscr{H}^{L}$.

Then also any connected Lie group containing $G_{E}(n) \otimes K$ as a subgroup does not admit a large compact subgroup.

4. We conjecture from these results that groups of the type $G=N \subset S$ do not admit a large compact subgroup unless $N$ is abelian and $S$ is compact.

Acknowledgments. The authors would like to thank Professor Abdus Salam, the International Atomic Energy Agency and UNESCO for hospitality at the International Centre for Theoretical Physics, Trieste. One of the authors (J. T.) is indebted to the A. von Humboldt Foundation for its support.

\section{References}

1. Mackey, G. W.: Infinite-dimensional group representations. Bull. Am. Math. Soc. 69, 628-686 (1963).

2. Harish-Chandra: Representations of semi-simple Lie groups I, II, III. Proc. Nat. Acad. Sci. (U.S.) 37, 170-173, 362-365, 366-369 (1951).

3. - Representations of semi-simple Lie groups I, II, III. Trans. Am. Math. Soc. 75, 185-243 (1953); 76, 26-65, 234-253 (1954).

4. Godement, R.: A theory of spherical functions I. Trans. Am. Math. Soc. 73, 496-556 (1952). 
5. Gel'fand,I. M., Graev, M.I., Pyatetskii-Shapiro, I.I.: Representation theory and automorphic functions, Vol. 6 of Generalized functions, Chap. I, Sec. 2.3. Philadelphia: W. B. Saunders Co. 1969.

6. Mackey,G.W.: The theory of induced representations. Lecture notes, (Chicago 1955) (mimeographed).

7. Smirnov, W.I.: Lehrgang der höheren Mathematik, Vol. V. Berlin: VEB Deutscher Verlag der Wissenschaften 1962.

8. Doebner, H.D., Melsheimer, O.: On limitable dynamical groups in quantum mechanics - I: General theory and spinless model. J. Math. Phys. 9, 1638-1656 (1968).

9. Melsheimer, O.: A new Lie group for the scalar $S U(3)$-symmetric strong coupling theory. Nucl. Phys. B5, 479-491 (1968).

10. Stein,E.M.: A survey of representations of non-compact groups in: High-energy physics and elementary particles, p. 563-584 (Secs. 6 and 8) (IAEA, Vienna 1965).

11. Hausner, M., Schwartz, J.T.: Lie groups, Lie algebras. Part II.7. New York: Gordon and Breach Sci. Publ. 1968.

Dr. J. Tolar

Faculty of Nuclear Science

Czech Technical University

Prague

Czechoslovakia

Prof. Dr. H. D. Doebner

Institut für theoretische Physik

Technische Universität Clausthal

BRD 3392 Clausthal, Leibnizstraße

Germany 